OPEN ACCESS

Edited by:

Paul Croarkin,

Mayo Clinic, United States

Reviewed by:

Ruiyang Ge,

University of British Columbia,

Canada

Xiangyu Long,

University of Calgary, Canada

Zhen Yuan,

University of Macau, China

${ }^{*}$ Correspondence:

Hang Zhang

kevinhangbnu@foxmail.com

Specialty section:

This article was submitted to Neuroimaging and Stimulation,

a section of the journal

Frontiers in Psychiatry

Received: 21 April 2019

Accepted: 27 August 2019

Published: 27 September 2019

Citation:

Zhou Z-W, Fang Y-T, Lan X-Q, Sun L,

Cao Q-J, Wang Y-F, Luo H, Zang Y-F

and Zhang $H$ (2019) Inconsistency

in Abnormal Functional Connectivity

Across Datasets of ADHD-200

in Children With Attention Deficit

Hyperactivity Disorder.

Front. Psychiatry 10:692.

doi: 10.3389/fpsyt.2019.00692

\section{Inconsistency in Abnormal} Functional Connectivity Across Datasets of ADHD-200 in Children With Attention Deficit Hyperactivity Disorder

\author{
Zhi-Wei Zhou 1,2,3, Yan-Tong Fang' ${ }^{2}$ Xia-Qing Lan 1,2,3, Li Sun ${ }^{4}$, Qing-Jiu Cao 4 , \\ Yu-Feng Wang 1,4, Hong Luo 1,2, Yu-Feng Zang ${ }^{1,2,3}$ and Hang Zhang ${ }^{1,2,3 *}$ \\ ${ }^{1}$ Institute of Psychological Sciences, College of Education, Hangzhou Normal University, Hangzhou, China, ${ }^{2}$ Center for \\ Cognition and Brain Disorders and the Affiliated Hospital, Hangzhou Normal University, Hangzhou, China, ${ }^{3}$ Zhejiang Key \\ Laboratory for Research in Assessment of Cognitive Impairments, Hangzhou, China, ${ }^{4}$ Institute of Mental Health, The Sixth \\ Hospital, Peking University, Beijing, China
}

Many studies have shown abnormal functional connectivity in children with attention deficit hyperactivity disorder (ADHD) by using resting-state functional magnetic resonance imaging (rs-fMRI). However, few studies illustrated that to what extent these findings were consistent across different datasets. The present study aimed to assess the consistency of abnormal functional connectivity in children with ADHD across the four datasets from a public-assess rs-fMRI ADHD cohort, namely, ADHD-200. We employed the identical analysis process of previous studies and examined a few factors, including connectivity with the seed regions of the bilateral dorsal anterior cingulate cortex, bilateral inferior frontal gyrus, and bilateral middle frontal gyrus; connectivity between default mode network and executive control network; stringent and lenient statistical thresholds; and the ADHD subtypes. Our results revealed a high inconsistency of abnormal seed-based connectivity in children with ADHD across all datasets, even across three datasets from the same research site. This inconsistency could also be observed with a lenient statistical threshold. Besides, each dataset did not show abnormal connectivity between default mode network and executive control network for ADHD, albeit this abnormal connectivity between networks was intensively reported in previous studies. Importantly, the ADHD combined subtype showed greater consistency than did the inattention subtype. These findings provided methodological insights into the studies on spontaneous brain activity of $A D H D$, and the ADHD subtypes deserve more attention in future studies.

Keywords: attention deficit hyperactivity disorder, resting-state fMRI, multi-site dataset, ADHD-200, functional connectivity

\section{INTRODUCTION}

Attention deficit hyperactivity disorder (ADHD) is one of the most common neurodevelopmental disorders in children (1). It is a highly heterogeneous disease characterized by varying degrees of inattention, hyperactivity, and impulsivity $(2,3)$. The pathogenesis of ADHD is incompletely understood, and a promising trend is the application of resting-state functional magnetic resonance 
imaging (rs-fMRI). rs-fMRI, measuring spontaneous brain activity, is easy to be implemented. It provides a consistent approach for clinical investigations; and two major measurements, that is, voxel-wise metrics and functional connectivity, were pervasively used in the rs-fMRI investigations on ADHD.

Voxel-wise metrics mainly include amplitude of low-frequency fluctuation (ALFF) (4), regional homogeneity (ReHo) (5), and degree centrality (DC) (6). The analytic process of the three metrics is similar across studies, thus helping to identify critical regions related to ADHD across fMRI studies $(7,8)$. By using these metrics, abnormal activity for ADHD was identified. As compared with typical developing children (TDC), ADHD showed decreased ALFF and ReHo in the brain areas of the right inferior frontal gyrus (rIFG), sensorimotor cortex, and anterior cingulate cortex $(4,9,10)$. Decreased DC for ADHD was observed in the bilateral pallidum (11). These results were usually acquired based on the data from a single dataset. Recently, our research group further examined these results on multiple datasets. Abnormal brain activities in frontalstriatal areas and frontal-parietal areas were identified through the metrics of ALFF, ReHo, and DC. Notably, none of the three metrics showed consistent results across the multiple datasets, even with a lenient threshold ( $p<0.05$, cluster size $>10$ voxels) (12). These findings suggest that it is necessary to re-assess the results of abnormal spontaneous brain activity of ADHD.

Functional connectivity was one another widely used measurement in the rs-fMRI studies on ADHD. Functional connectivity was defined as the correlation between the time course of a particular brain region (seed region) and all other voxels in the brain (13), and it provides detailed information about interregional relationships. In the functional connectivity explorations, three frontal regions (dorsal anterior cingulate cortex (dACC), right middle frontal gyrus (rMFG), and rIFG) identified by Weissman et al. (14) were believed to be important for the presence of ADHD (14). The three regions exhibited negative connectivity with precuneus and posterior cingulate cortex (PCC), and ADHD adults showed decreased functional connectivity between dACC and precuneus/PCC than did TDC (15). This finding was further reproduced by an investigation on ADHD children (16), and it was also believed as one biomarker of ADHD (17). Aside from the seed-based functional connectivity, the connectivity between the default mode network (DMN) and executive control network (ECN) was discussed extensively. ECN and DMN tend to be active or inactive during cognitively demanding tasks (18). DMN consists of anterior and medial prefrontal cortex (PFC), the precuneus, and the angular gyrus $(19,20)$. It is active when individuals are engaged in internally focused tasks including autobiographical memory retrieval, envisioning the future, and conceiving the perspectives of others (21). ECN mainly includes lateral PFC, dorsal parietal cortex, sensorimotor cortex, subcortical areas, and the cerebellum $(19,22)$. This network is usually activated during the performance of externally oriented tasks, so it is also termed as task-positive network (23). ECN and DMN show negative and positive connectivity with PCC $(18,24,25)$. The negative connectivity between the two networks has been widely reported, and clinical evidences indicated the methylphenidate improves the symptoms of ADHD and meanwhile increases the negative connectivity between ECN and DMN (23).
The seed-based functional connectivity and connectivity between ECN and DMN inform our understanding about the pathological mechanism of ADHD. However, to what extent the functional connectivity results are consistent across individual datasets remains unclear. To address this issue, the present study examined the abnormal functional connectivity based on the datasets of ADHD200. ADHD-200 is one of the most widely used multi-site MRI cohorts of $\mathrm{ADHD}$, and it contains 10 independent datasets from eight different sites (26). These datasets provide rs-fMRI and anatomical MRI data of both ADHD and TDC. The consistency of seed-based functional connectivity and the negative connectivity between ECN and DMN were first examined across individual datasets. Moreover, ADHD involves three subtypes, that is, inattention, hyperactivity/ impulsivity, and combined (27). It was observed that children with different $\mathrm{ADHD}$ subtypes showed differences in spontaneous brain activity $(28,29)$. Thus, a single subtype may show greater consistency of abnormal spontaneous brain activity than the mixed subtypes of ADHD. Therefore, analyses based on the subtypes were also involved in the present study.

\section{METHODS AND MATERIALS}

\section{Participants and Data Acquisition}

The data we used in this study are publicly available from the ADHD-200 Consortium (http://fcon_1000.projects.nitrc.org/ indi/adhd200). The ADHD-200 cohort contains both functional and anatomical MRI data contributed by eight institutions. Each dataset was approved by the research ethics review boards of each institution. Signed informed consent was obtained from all participants or their legal guardian before participation.

The datasets were first selected according to the following criteria (Figure 1A): (1) Including both ADHD and TDC groups; so the data from BU, University of Pittsburgh, and Washington University were excluded. (2) Employing the same time of repetition (TR) of $\leq 2,000 \mathrm{~ms}$ across the datasets. According to this criterion, Kennedy Krieger Institute (KKI) $(\mathrm{TR}=2,500 \mathrm{~ms})$ and Oregon Health \& Science University (OHSU) (TR $=2,500 \mathrm{~ms}$ ) were excluded. Then, the datasets of NYU, PKU1, PKU2, and PKU3 were included in the study, and data from NeuroImage $(\mathrm{TR}=1,960 \mathrm{~ms}$ ) were also excluded because the TR of NeuroImage was not the same as the TR of the other four datasets. The PKU2 and PKU3 datasets only had male subjects, so the female subjects in NYU and PKU1 datasets were excluded to remove potential confounding effect of gender on the results. Left-handed subjects were also excluded from each dataset. There is no significant difference in age between children with $\mathrm{ADHD}$ and TDC across all datasets in the present study. After case-by-case age matching between ADHD and TDC, 58 subjects from NYU, 30 from PKU1, 56 from PKU2, and 38 from PKU3 were included in the current study. Demographic information of all subjects is summarized in Table $\mathbf{1}$.

Medications were withheld for at least $24 \mathrm{~h}$ prior to scanning. More detailed demographic characteristics of the participants of the four datasets can be seen in http://fcon_1000.projects.nitrc. org/indi/adhd200. The rs-fMRI data of the four datasets were acquired from three scanners, with TR of 2,000 ms for all. PKU1 and PKU2 used the same scanner, but scanning parameters 
A

Including both ADHD and TDC group.

Employing the same TR with less than or equal to $2000 \mathrm{~ms}$.

Including only male subjects.

Including only right-handness subjects.

Age matching between ADHD and TDC.
B

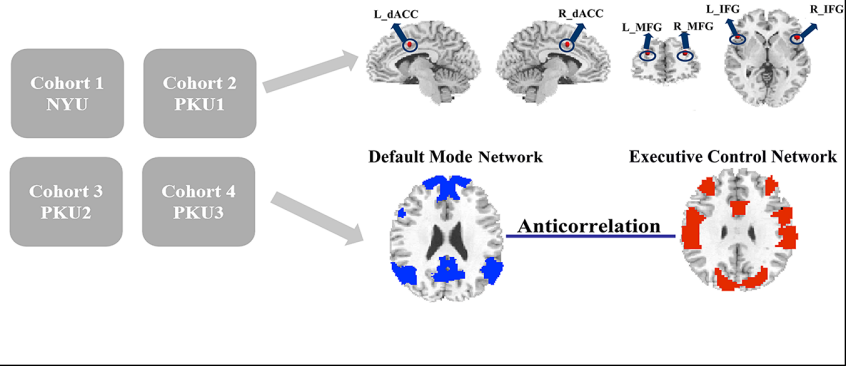

FIGURE 1 | Flowchart of date exclusion (A) and data analysis (B).

TABLE 1 | Demographic information of each dataset in current study.

\begin{tabular}{|c|c|c|c|c|c|c|c|c|}
\hline & \multicolumn{2}{|c|}{ NYU } & \multicolumn{2}{|c|}{ PKU1 } & \multicolumn{2}{|c|}{ PKU2 } & \multicolumn{2}{|c|}{ PKU3 } \\
\hline & ADHD & TDC & ADHD & TDC & ADHD & TDC & ADHD & TDC \\
\hline$N$ & 29 & 29 & 15 & 15 & 28 & 28 & 19 & 19 \\
\hline Gender (male) & 29 & 29 & 15 & 15 & 28 & 28 & 19 & 19 \\
\hline Age (year) & $12.1 \pm 2.9$ & $12.2 \pm 2.8$ & $11.2 \pm 2.3$ & $11.6 \pm 1.5$ & $12.7 \pm 1.7$ & $11.7 \pm 1.8$ & $13.2 \pm 1.3$ & $13.3 \pm 1.0$ \\
\hline$I Q$ & $106 \pm 16.0$ & $115.3 \pm 14.3$ & $101.7 \pm 12.4$ & $123.0 \pm 14.2$ & $111.5 \pm 12.7$ & $121.6 \pm 12.2$ & $102.7 \pm 10.4$ & $111.7 \pm 12.7$ \\
\hline Subtype & 19/10/0 & - & $6 / 9 / 0$ & - & 12/16/0 & - & 7/12/0 & - \\
\hline
\end{tabular}

Data are presented as mean \pm SD. C, ADHD-combined; I, ADHD-inattention; H, ADHD-hyperactive/impulsive. ADHD, attention deficit hyperactivity disorder; TDC, typical

developing children.

were slightly different. The detailed parameters are listed in Supplementary Material Table S1.

\section{Data Processing}

The preprocessing was carried out using the Data Processing Assistant for Resting-State fMRI (DPARSF) (30), which is based on the Statistical Parametric Mapping (SPM8) (http:// www.fil.ion.ucl.ac.uk/spm) and Resting-State fMRI Data Analysis Toolkit (REST) (31) (http://www.restfmri.net). The first 10 time points were removed for signal stabilization and participant adaptation. And then, the number of left time points for NYU, PKU1, PKU2, and PKU3 is 170, 230, 230, and 230, respectively. The unified number of time points (first 170 volumes) was employed for all of the four datasets. Slice timing correction and image realignment to correct head motion were followed. The head motion criteria, that is, head motion $<3 \mathrm{~mm}$ translation or $<3^{\circ}$ rotation in any direction, were employed, referring to previous studies (32-34), and all subjects met these criteria. Individual structural images were segmented after co-registered to functional images. Then, functional images were spatial normalized to Montreal Neurological Institute (MNI) template (re-sampled into $3 \times$ $3 \times 3 \mathrm{~mm}^{3}$ ) and smoothed with an $6 \times 6 \times 6$ full-width-athalf-maximum (FWHM) Gaussian kernel. The head motion parameter measured by Friston-24 model, global signal effect, white matter (WM), and cerebrospinal fluid (CSF) signals were further regressed out as nuisance covariates. The time course of each voxel was linearly detrended and band-pass filtered with frequencies ranging from 0.01 to $0.08 \mathrm{~Hz}$.

\section{Seed-Based Functional Connectivity Analyses}

The functional connectivity analyses were performed based on the seed regions of interest (ROIs), including R_dACC, R_IFG, and R_ MFG. The three seed regions were defined as a sphere with a 6-mm radius (34 voxels) centered at the coordinates reported by Weissman et al. (14) converted to MNI space (R_dACC: $x=8, y=7, z=38$; R_ IFG: $x=34, y=45, z=23$; R_MFG: $x=49, y=19, z=0)$. Moreover, asymmetry of the human brain was mentioned intensively (35, 36), so we also performed the functional connectivity analyses in the contralateral ROIS, and the coordinates were converted to MNI space (L_dACC: $x=-8, y=7, z=38$; L_IFG: $x=-34, y=45, z=23$; L_MFG: $x=-49, y=19, z=0$ ). The mean time course of each seed region was extracted as reference time course. Then, the functional connectivity was calculated as Fisher's Z-transformed Pearson correlation coefficient between the reference time course and the time course of each voxel in the brain.

\section{Connectivity Between ECN and DMN}

A public DMN mask created by Yeo et al. (37) was employed in the analysis (37), and regions showing time course negative correlation with the DMN were identified as ECN $(p<0.001$, Gaussian random field (GRF) corrected). Then, the mean time courses were extracted based on the ECN and DMN masks in each subject. These masks were unified for the subjects of all the datasets, and therefore, there was no network difference across the datasets. The network connectivity was further calculated through the Pearson correlation between the mean time course of DMN and the mean time course of ECN. 
To validate the analysis with public network masks, we performed another network construction analysis. In accordance with previous studies $(38,39)$, DMN/ECN was identified as regions showing positive/negative time course correlation with PCC $(p<0.001$, GRF corrected $)$, and the networks were constructed for each dataset. Functional connectivity with the seed region of PCC was calculated [6-mm spherical ROI centered at MNI coordinates: $x=1$, $y=-55, z=17$, reported by Vincent et al. (40)] (see details in Supplementary Materials 2.6). Then, the connectivity between ECN and DMN was re-calculated and compared between ADHD group and control group in each dataset. Since the networks were constructed for each dataset, the similarities and differences of the networks across all of the four datasets were further examined by the measurement of percentage of overlap (Figure S40 and Table S4). Moreover, the effects of various analytical methods on the constructed networks were further assessed by the measurement of the Dice coefficient (Figure S41 and Table S5).

\section{Statistical Analysis}

Functional connectivity maps of the seed regions of R_dACC, R_IFG, R_MFG, L_dACC, L_IFG, and L_MFG were compared between the groups of children with ADHD and TDC. Twosample $t$-tests were performed on each dataset. The comparison result for each dataset was corrected for multiple comparisons $(p<0.005$, GRF corrected)

At the same time, to reduce the possibility of false-negative results, a more lenient threshold $(p<0.05$, cluster size $>10$ voxels) was also used for each dataset.

We also performed the analyses of standardized effect size (SES) based on Cohen's $d$, which is calculated in the following equation (41):

$$
\begin{gathered}
\text { Cohen's } d=\frac{\bar{X}_{A D H D}-\bar{X}_{A D H D}}{S_{A L L}} \\
S_{A L L}=\sqrt{\frac{\left(n_{A D H D}-1\right) S_{A D H D}^{2}+\left(n_{T D C}-1\right) S_{T D C}^{2}}{n_{A D H D}+n_{T D C}-2}}
\end{gathered}
$$

According to equation of independent two-sample $t$-test, as follows,

$$
t=\frac{\bar{X}_{A D H D}-\bar{X}_{T D C}}{\sqrt{\frac{\left(\mathrm{n}_{A D H D}-1\right) S_{A D H D}^{2}+\left(\mathrm{n}_{T D C}-1\right) S_{T D C}^{2}}{\mathrm{n}_{A D H D}+\mathrm{n}_{T D C}-2}\left(\frac{1}{\mathrm{n}_{A D H D}}+\frac{1}{\mathrm{n}_{T D C}}\right)}}
$$

The relationship of Cohen's $d$ and $t$-value can be obtained, as follows:

$$
\text { Cohen's } d=t \sqrt{\frac{n_{A D H D}+n_{T D C}}{n_{A D H D} \cdot n_{T D C}}}
$$

According to Equation (3), we transformed $t$ maps into SES map for each dataset. The same threshold was applied to the SES maps of each dataset. A high level SES of 0.8 was used, which corresponded to $t=3.08,2.16,2.96$, and $2.50(p=0.003,0.039$, 0.005 , and 0.017 ) for NYU, PKU1, PKU2, and PKU3, respectively.

To view the consistency of results, the thresholded $t$ maps and SES maps were binarized and overlapped among the four datasets. The number of overlapped voxels across four and three datasets was quantified using the Dice overlap coefficient (42), where the voxel number of intersection was divided by the total voxel number of all the datasets.

As for the connectivity between ECN and DMN, the individual correlation coefficient between the time courses of two networks was transformed by Fisher' $Z$ transformation. Then, a twosample $t$-test of the Fisher' $Z$-transformed correlation coefficients was performed between the ADHD group and TDC group in each dataset.

\section{Analysis on Subtypes}

In the current study, there were two ADHD subtypes in all of the four datasets, that is, the ADHD combined subtype and the ADHD inattention subtype. The information on subtypes was provided by ADHD-200 directly. According to the diagnostics illustration provided by ADHD-200 (http://fcon_1000.projects.nitrc.org/indi/ adhd200/), PKU1, PKU2, and PKU3 used the ADHD Rating Scale (ADHD-RS) IV to determine the ADHD subtypes, and NYU used Conners' Parent Rating Scale-Revised, long version (CPRS-LV), to identify the ADHD subtypes. There were $19,6,12$, and 7 participants with the ADHD combined subtype, and 10, 9, 16, and 12 participants with the ADHD inattention subtype in NYU, PKU1, PKU2, and PKU3, respectively. Each subtype matched the health control with age. All the above analysis procedures were repeated based on the data of each ADHD subtype.

\section{RESULTS}

\section{Seed-Based Functional Connectivity Across Datasets}

Abnormal seed-based functional connectivity examined in each dataset is shown in Supplementary Material Figures S1-S6, and the overlapped results across the four datasets for each seed region are shown in Figure 2. No overlapped abnormal functional connectivity was observed from three or four datasets. Even using a more lenient threshold, several voxels showed overlapped abnormal functional connectivity from three or four datasets. Using R_dACC as the seed, we observed seven overlapped voxels of NYU, PKU1, and PKU3 in the left cerebellum. Using R_MFG as the seed, we observed that 13 voxels overlapped from NYU, PKU1, and PKU3 in the PCC. When using L_dACC as the seed, we observed 26 overlapped voxels of NYU, PKU1, and PKU2 in the right medial orbital frontal gyrus and 16 voxels in the left paracentral lobule (see details in Figure 3 and Table 2). 

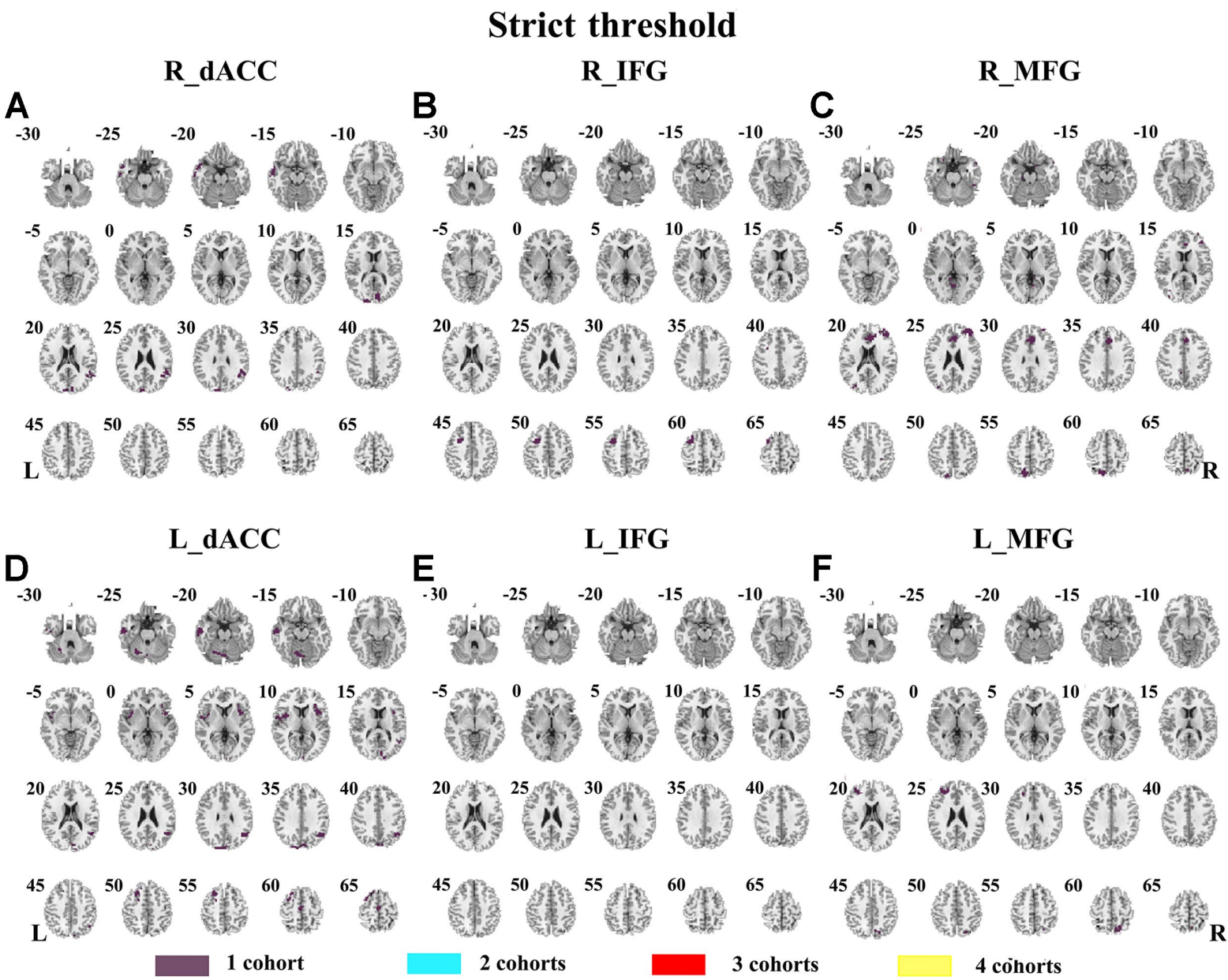

FIGURE 2 | The overlapped results of abnormal functional connectivity for ADHD across the four datasets. The functional connectivity was assessed with the seed regions of $R \_d A C C, R \_I F G, R \_M F G, L \_d A C C, L \_I F G$, and $L \_M F G$. (A-F) indicate the results with stringent $(p<0.005$, GRF corrected). Purple indicates the regions detected in only one of the four datasets. Mint, red, and yellow indicate the regions detected in two, three, and four datasets, respectively. ADHD, attention deficit hyperactivity disorder; dACC, dorsal anterior cingulate cortex; IFG, inferior frontal gyrus; MFG, middle frontal gyrus; GRF, Gaussian random field.

The SES maps of each dataset are shown in Supplementary Material Figures S7-S12, and the overlapped SES maps across datasets are shown in Figure 4 (with SES > 0.8). No clusters showed overlaps from more than three datasets.

The seed-based functional connectivity was further examined in subtypes of ADHD, including the combined subtype and the inattention subtype. Abnormal functional connectivity of each dataset is shown in Supplementary Material Figures S13-S24. For the combined subtype, abnormal connectivity between R_IFG and middle frontal gyrus showed consistency across all datasets. This consistency was not identified in the inattention subtype (Figures $\mathbf{5}$ and 6 and Table 3).

The SES maps of each dataset/each ADHD subtype are shown in Supplementary Material Figures S25-S36. For each ADHD subtype, the overlapped SES maps across all datasets are shown in Figures 7 and 8. Abnormal functional connectivity of each seed region showed overlaps from more than three datasets, and these overlaps were only observed in the combined subtype but not in the inattention subtype.

Considering that the global signal effect on the functional connectivity analysis is still controversial, we also repeated the above analysis based on the preprocessed data without removing the global signal effect. The results are shown in the Supplementary Materials (Figures S42-S48), which also exhibited few overlaps of the seed-based functional connectivity across the four datasets.

\section{Connectivity Between ECN and DMN}

Both children with ADHD and TDC in each dataset showed strong negative connectivity between ECN and DMN (Figure 9). No significant difference of this negative connectivity was observed between children with ADHD and TDC in each dataset (Table 4). This finding was reproduced by our further validation analysis 


\section{Lenient threshold}

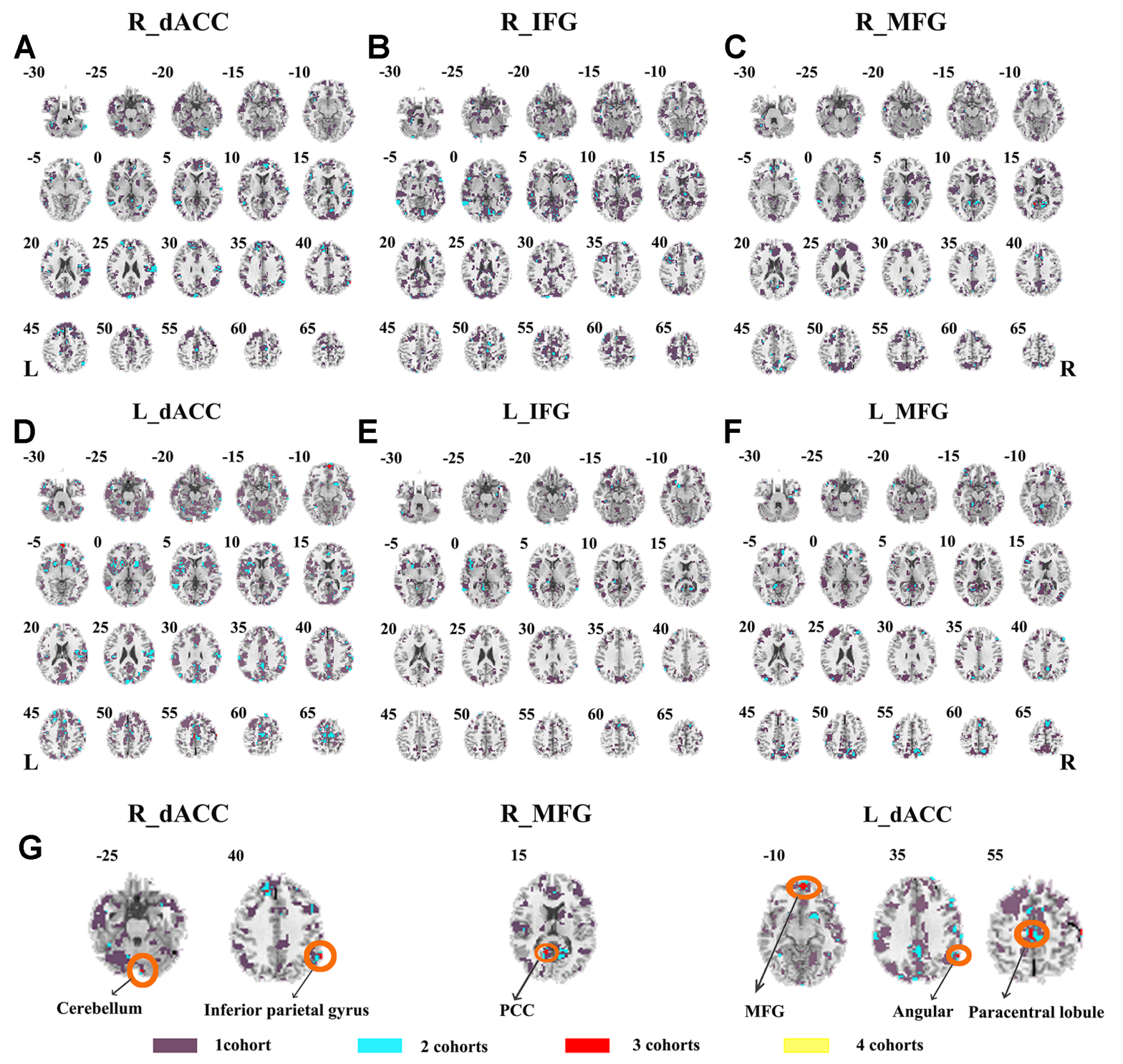

FIGURE 3 | The overlapped results of abnormal functional connectivity for ADHD across the four datasets. The functional connectivity was assessed with the seed regions of $R \_d A C C$, R_IFG, R_MFG, $L \_d A C C, L \_I F G$, and $L \_M F G$. (A-G) indicate the results with lenient $(p<0.05$, cluster size $>10)$ thresholds. Purple indicates the regions detected in only one of the four datasets. Mint, red, and yellow indicate the regions detected in two, three, and four datasets, respectively. ADHD, attention deficit hyperactivity disorder; dACC, dorsal anterior cingulate cortex; IFG, inferior frontal gyrus; MFG, middle frontal gyrus.

TABLE 2 | Clusters showing overlaps for three/four datasets and contained maximal of overlapped voxels.

\begin{tabular}{|c|c|c|c|c|c|c|}
\hline Seed & Number of overlapped cohorts & Region & L/R & BA & Number of overlapped voxels & Dice \\
\hline \multirow[t]{2}{*}{ R_dACC } & 3 & Cerebellum & $\mathrm{R}$ & - & 2 & 0.0002 \\
\hline & & Inf. parietal gyrus & $\mathrm{R}$ & 40 & 4 & 0.0003 \\
\hline R_MFG & 3 & Post. cingulate cortex & $\mathrm{L}$ & 29 & 13 & 0.0050 \\
\hline & & Angular & $\mathrm{R}$ & 40 & 5 & 0.0011 \\
\hline & & Paracentral lobule & $\mathrm{L}$ & 6 & 16 & 0.0034 \\
\hline
\end{tabular}

Med., medial; Inf., inferior; Post., posterior; L, left; R, right; dACC, dorsal anterior cingulate cortex; IFG, inferior frontal gyrus; MFG, middle frontal gyrus. The threshold was $p<0.05$, and cluster size $>10$ voxels for each cohort. 

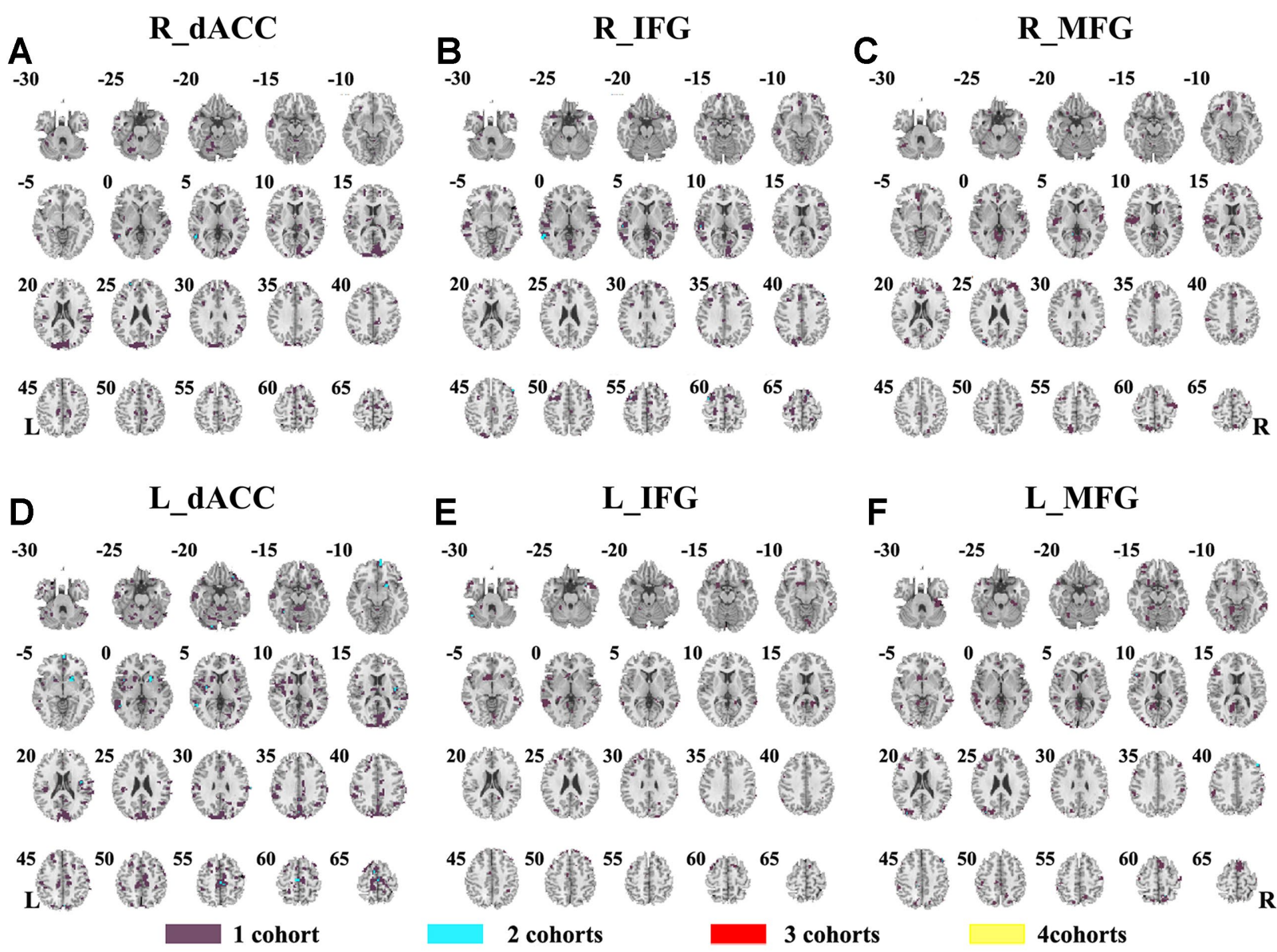

2 cohorts

3 cohorts

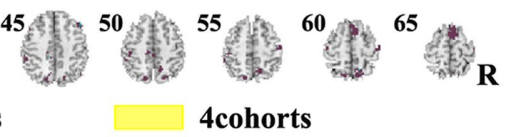

FIGURE 4 | The overlapped effect size results of the individual dataset. The threshold of effect size was set at 0.8 for each dataset. (A-F) indicate the results by using R_dACC, R_IFG, R_MFG, $L_{-} d A C C, L_{-} I F G$, and $L_{-} M F G$ as the seed regions, respectively. Purple indicates the regions detected only in one of the four datasets. Mint indicates the regions detected in two datasets. Red indicates the regions detected by only three datasets. Yellow indicates the regions detected in four datasets. dACC, dorsal anterior cingulate cortex; IFG, inferior frontal gyrus; MFG, middle frontal gyrus.

with another network construction method (see details in Supplementary Material Figure S37 and Table S2).

We also analyzed the negative connectivity between ECN and $\mathrm{DMN}$ in ADHD subtypes. As Figure 10 shows, this negative connectivity in each dataset did not show significant difference between the children with the inattention subtype and TDC. Children with the combined subtype showed the trend of significant difference between ADHD and TDC in PKU2, $t(22)=$ 2.69, $p=0.01$ (Figure 10 and Table 5); however, these results could not survive after the multiple comparison correction.

These results could be reproduced in our validation analysis with another network construction method (details in Supplementary Material Figures S38 and S39 and Table S3). However, we failed to identify the negative connectivity between ECN and DMN when reproducing the results based on the preprocessed data without removing the global signal effect (Supplementary Material Figures S49-S54 and Tables S6-S9).

\section{DISCUSSION}

The current study examined the consistency of abnormal functional connectivity across datasets of ADHD-200. We employed seedbased functional connectivity, the negative connectivity between ECN and DMN, stringent and lenient statistical thresholds, and ADHD subtypes in the analysis process. Three major results were obtained: (1) The abnormal seed-based functional connectivity of ADHD was not consistent across datasets. (2) For each dataset, the negative connectivity between ECN and DMN did not show any significant difference between ADHD and TDC. (3) In a subtype analysis, the combined subtype showed more consistent results across datasets as compared with the inattention subtype.

As previous studies reported, functional connectivity between PCC/precuneus and the three regions of R_dACC, R_IFG, and R_MFG could be observed in children with ADHD and TDC (15, 43 ), and the decreased functional connectivity between dACC and $\mathrm{PCC} /$ precuneus was intensively reported in rs-fMRI studies on 

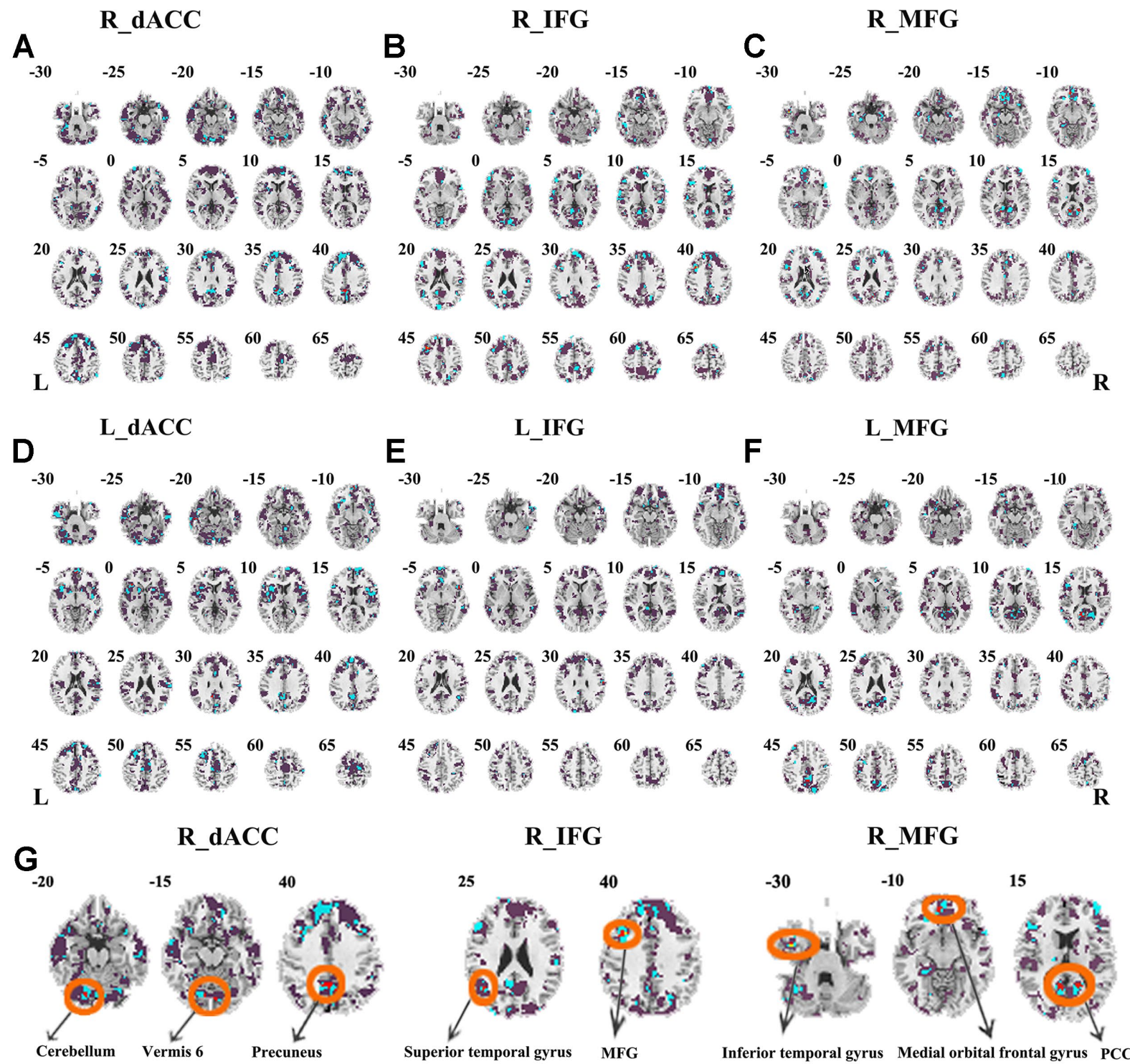

L_dACC
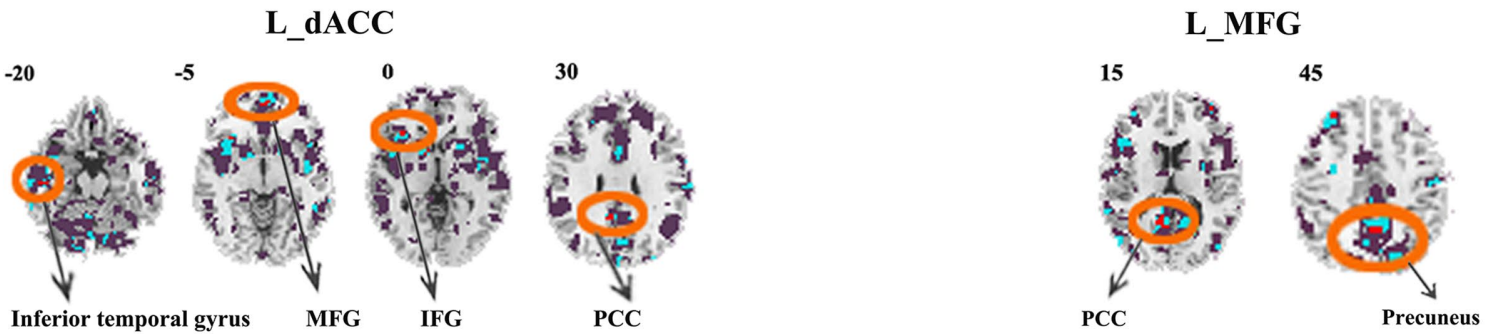

1 cohort

2 cohorts

3 cohorts

4 cohorts

FIGURE 5 | The overlapped results across the four datasets in the ADHD combined subtype. (A-G) indicate the results detected by using R_dACC, R_IFG, R_ MFG, L_dACC, L_IFG, and L_MFG as the seed regions. Purple indicates regions detected in only one of the four datasets. Mint, red, and yellow indicate the regions detected in two, three, and four datasets, respectively. ADHD, attention deficit hyperactivity disorder; $\mathrm{AACC}$, dorsal anterior cingulate cortex; IFG, inferior frontal gyrus; MFG, middle frontal gyrus. 


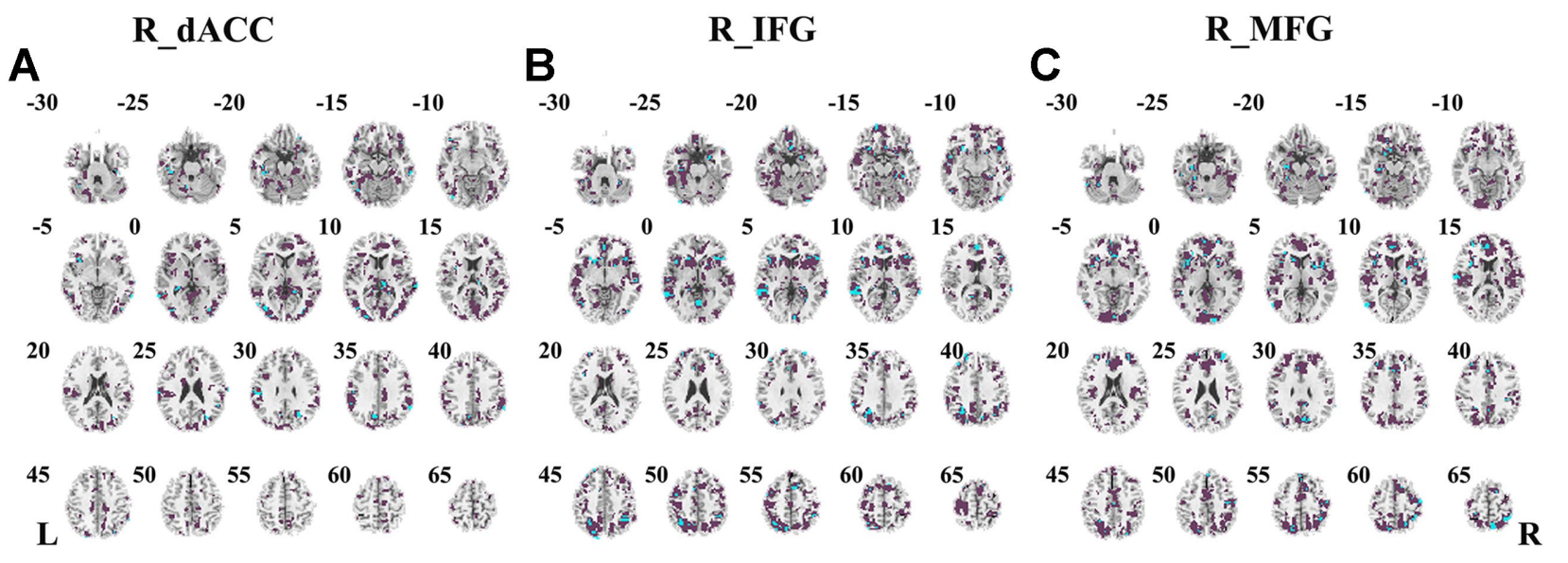

\section{L dACC}

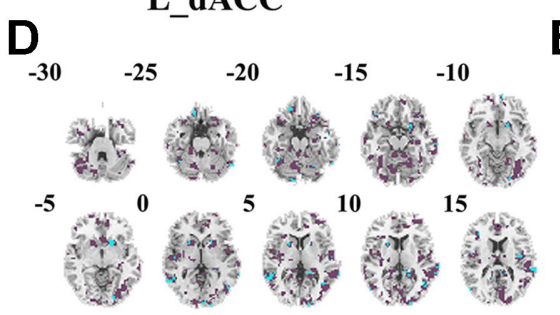

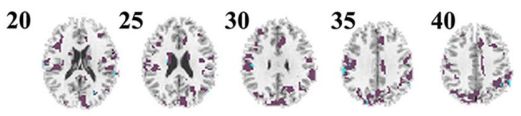

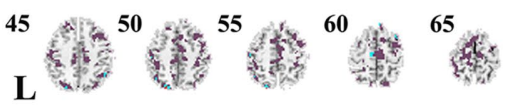

R_dACC

G

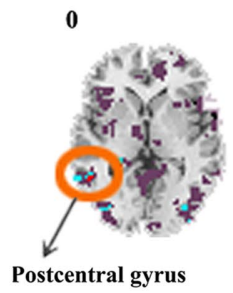

E L_IFG

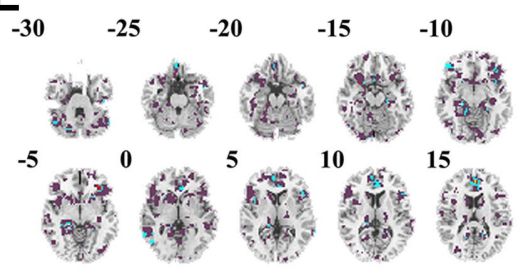

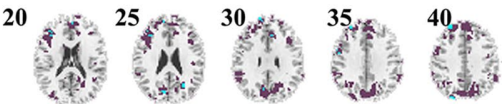

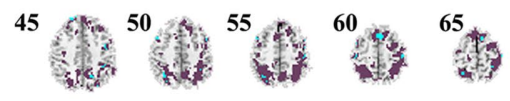

$\mathbf{F}$

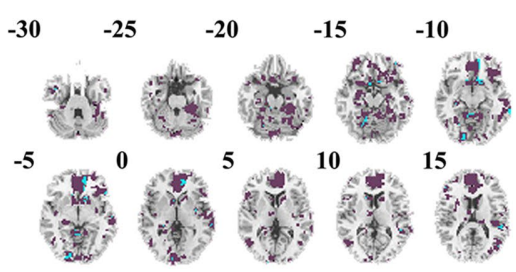

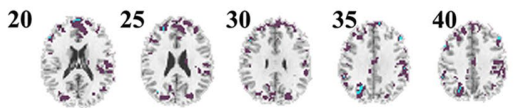

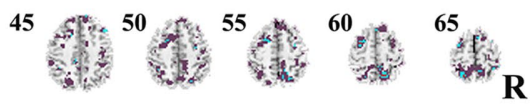

L_IFG
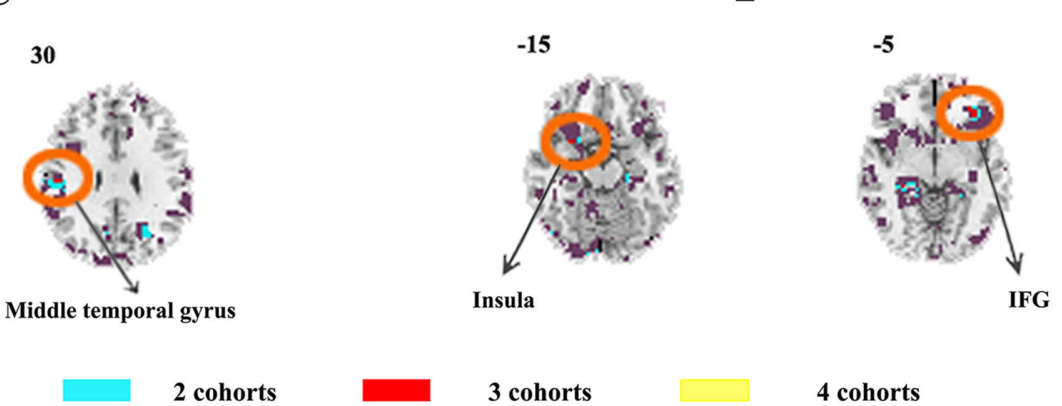

3 cohorts

4 cohorts

FIGURE 6 | The overlapped results across the four datasets in the ADHD inattention subtypes. (A-G) indicate the results detected by using $R \_d A C C$, $R$ _IFG, $R$ _ MFG, L_dACC, L_IFG, and L_MFG as the seed regions. Purple indicates regions detected in only one of the four datasets. Mint, red, and yellow indicate the regions detected in two, three, and four datasets, respectively. ADHD, attention deficit hyperactivity disorder; dACC, dorsal anterior cingulate cortex; IFG, inferior frontal gyrus; MFG, middle frontal gyrus.

$\operatorname{ADHD}(16,44,45)$. In the present study, this abnormal functional connectivity was not consistent across all datasets. Decreased connectivity between R_dACC and PCC/precuneus was observed only in the datasets of NYU and PKU1. The abnormal functional connectivity showed some overlaps from three datasets in the region of right inferior parietal gyrus, right supplementary motor area (SMA), and so on. However, the highest Dice overlap coefficient is just 0.0057 even when using a lenient statistical threshold $(p<$ 0.05 , cluster size $>10$ voxels). Notably, the inconsistency could also be observed in three datasets from the same research site, PKU. Furthermore, in each dataset, the SES of the abnormal connectivity was small, and no overlaps of the SES maps were observed from more than three datasets. These findings suggest high inconsistency of abnormal functional connectivity in children with ADHD, and 
TABLE 3 | Clusters that were the overlap for three/four datasets and contained maximal of overlapped voxels.

\begin{tabular}{|c|c|c|c|c|c|c|}
\hline Seed & Number of overlapped cohorts & Region & L/R & BA & Number of overlapped voxels & Dice \\
\hline \multicolumn{7}{|c|}{ ADHD combined subtype } \\
\hline \multirow[t]{3}{*}{ R_dACC } & 3 & Cerebellum & $\mathrm{L}$ & - & 14 & 0.0035 \\
\hline & & Vermis 6 & - & - & 7 & 0.0006 \\
\hline & & Precuneus & $\mathrm{L}$ & 7 & 14 & 0.0032 \\
\hline \multirow[t]{3}{*}{ R_IFG } & 4 & Mid. frontal gyrus & $\mathrm{L}$ & 9 & 5 & 0.0015 \\
\hline & 3 & Sup. temporal gyrus & $\mathrm{L}$ & 9 & 9 & 0.0029 \\
\hline & & Mid. frontal gyrus & $\mathrm{L}$ & 9 & 46 & 0.0011 \\
\hline \multirow[t]{3}{*}{ R_MFG } & 4 & Inf. temporal gyrus & $\mathrm{L}$ & 20 & 2 & 0.0008 \\
\hline & 3 & Med. orbital frontal gyrus & $\mathrm{L}$ & 11 & 29 & 0.0101 \\
\hline & & Post. cingulate cortex & $\mathrm{L} / \mathrm{R}$ & 30 & 40 & 0.0046 \\
\hline \multirow[t]{4}{*}{ L_dACC } & 3 & Inf. temporal gyrus & $\mathrm{L}$ & 20 & 6 & 0.0016 \\
\hline & & Med. frontal gyrus & $\mathrm{L}$ & 11 & 13 & 0.0035 \\
\hline & & Inf. frontal gyrus & $\mathrm{L}$ & 45 & 9 & 0.0024 \\
\hline & & Post. cingulate cortex & $\mathrm{L}$ & 30 & 12 & 0.0029 \\
\hline \multirow[t]{2}{*}{ L_MFG } & 3 & Post. cingulate cortex & $\mathrm{L}$ & 30 & 16 & 0.0060 \\
\hline & & Precuneus & $\mathrm{R}$ & 7 & 8 & 0.0030 \\
\hline \multicolumn{7}{|c|}{ ADHD inattention subtype } \\
\hline \multirow[t]{2}{*}{ R_dACC } & 3 & Mid. temporal gyrus & L & 39 & 5 & 0.0008 \\
\hline & & Postcentral gyrus & $\mathrm{L}$ & 2 & 5 & 0.0023 \\
\hline \multirow[t]{2}{*}{ L_IFG } & 3 & Inf. frontal gyrus & $\mathrm{R}$ & 45 & 5 & 0.0020 \\
\hline & & Insula & $\mathrm{L}$ & 38 & 1 & 0.0001 \\
\hline
\end{tabular}

Med., medial; Mid., middle; Inf., inferior; Sup., superior; Post., posterior; L, left; R, right; dACC, dorsal anterior cingulate cortex; IFG, inferior frontal gyrus; MFG, middle frontal gyrus; $A D H D$, attention deficit hyperactivity disorder. The threshold was $p<0.05$, and cluster size $>10$ voxels.

such inconsistency may be related to the heterogeneity of ADHD $(2,46)$. It is important to further validate the findings of functional connectivity in children with ADHD, and at least, both statistical and SES results should be provided in future studies.

Negative connectivity between ECN and DMN was widely examined, and it was believed as the toggling between the executive state and introspective state $(25,47,48)$. In the present study, each subject showed strong negative connectivity between ECN and DMN. Such negative connectivity has attracted more attention from ADHD research communities for its correlation with the stability of behavior (49). Clinically, this negative connectivity was decreased in children with ADHD without medicine and was increased after use of methylphenidate (23). In the present study, no significant difference of this negative connectivity was identified between children with ADHD and TDC in each dataset. This finding could be validated by different network construction methods. The networks constructed by the data of individual dataset were different across all of four datasets (the percentage of common region from $51.67 \%$ to $66.60 \%$ ). Moreover, these networks showed difference of spatial pattern as compared with the networks provided by the previous study (37) (Dice coefficient from 0.58 to 0.63 ). The difference of network spatial pattern may affect the results of the connectivity between networks (50). Thus, we directly employed the networks of Yeo et al. (37) to further validate the negative functional connectivity between $\mathrm{ECN}$ and DMN, and the findings were not changed. Moreover, we observed the trend of significant difference when performing analysis in the ADHD subtypes. Children with the combined subtype showed the trend of significant difference between ADHD and TDC in PKU2; however, these results could not survive after the multiple comparison correction. So these results were not enough to support the biomarker role of this negative connectivity for the presence of ADHD.
The ADHD involve three subtypes, that is, inattention, hyperactivity/impulsivity, and combined (27). It was suggested that the mixed ADHD subtypes may have confounding effects on the results of abnormal spontaneous brain activity (29). Thus, ADHD subtype was considered as a factor in the analysis process. Here, only the combined subtype and the inattention subtype were involved in the datasets. Results from the combined subtype showed more consistency than did the inattention subtype, and combined subtype showed more overlapped regions across more than three datasets. Specifically, children with the combined subtype showed increased functional connectivity between R_IFG and middle frontal gyrus in all of four datasets. Moreover, considering that the sample size may affect the results, we further calculated the effect size. Also, with high SES (> 0.8), combined subtype exhibited overlapped regions from three datasets. These overlapped regions, such as IFG, MFG, precuneus, PCC, cerebellum, and SMA, have been reported by previous studies on ADHD. For example, Maarten et al. (51) found that increased positive connectivity between SMA and MFG was associated with inhibitory function (51). Duann et al. (52) also found that the greater connectivity between IFG and SMA is related to successful response inhibition (52). Rubia et al. (53) reported that the negative correlation in ADHD patients between reduced activation in PCC and hyperactivity scale scores confirms a relationship between behavioral neural abnormalities and impulsiveness (53). So these overlapped regions may be related to the impulsiveness and inhibition. By contrast, children with the inattention subtype did not show any overlaps of abnormal functional connectivity across more than three cohorts, considering that the ADHD combined subtype included symptoms of hyperactivity/impulsivity and inattention. Thus, the consistency of abnormal functional connectivity for ADHD combined subtype may be not related to inattention symptom in ADHD. 


\section{ADHD combined subtype}
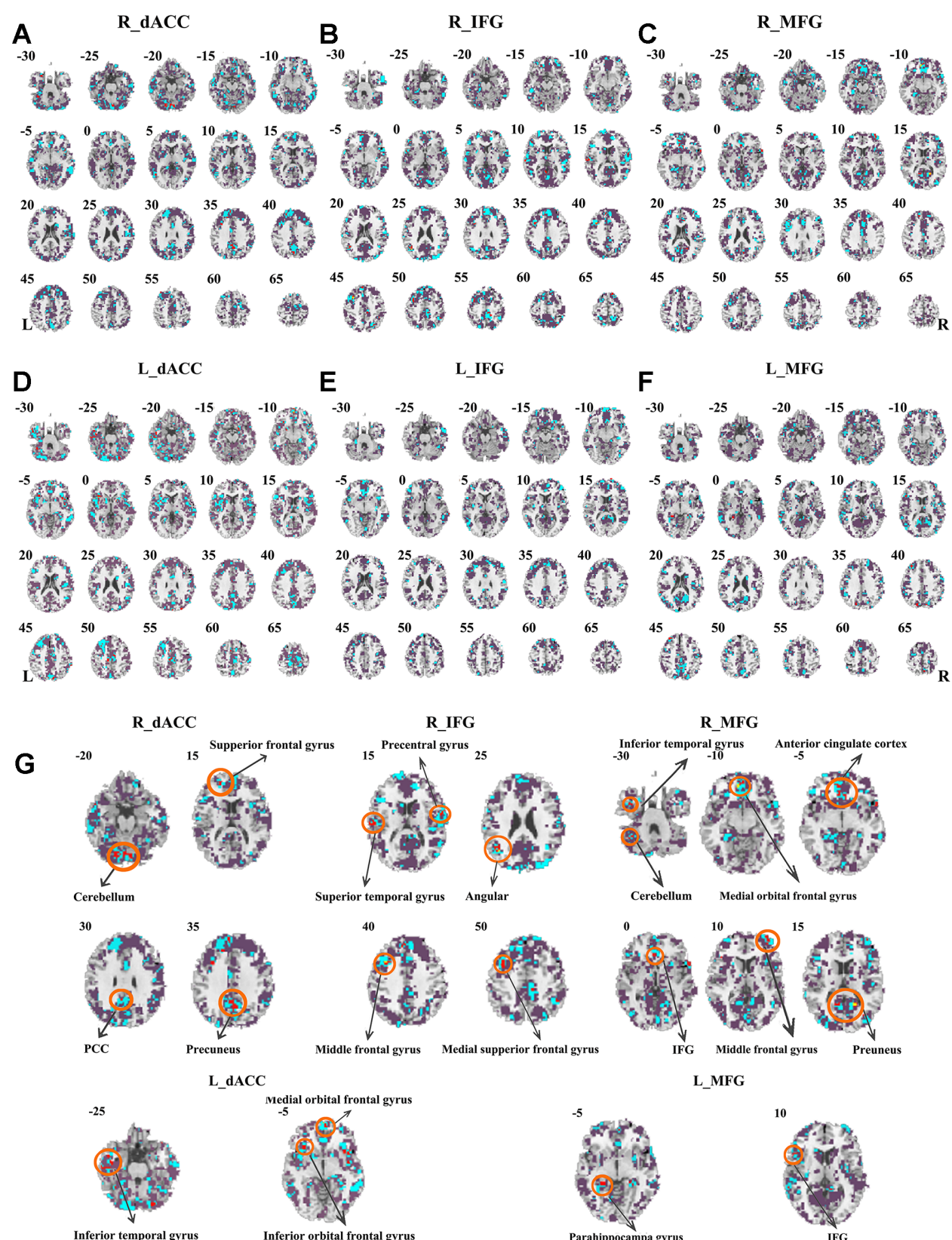

L_dACC
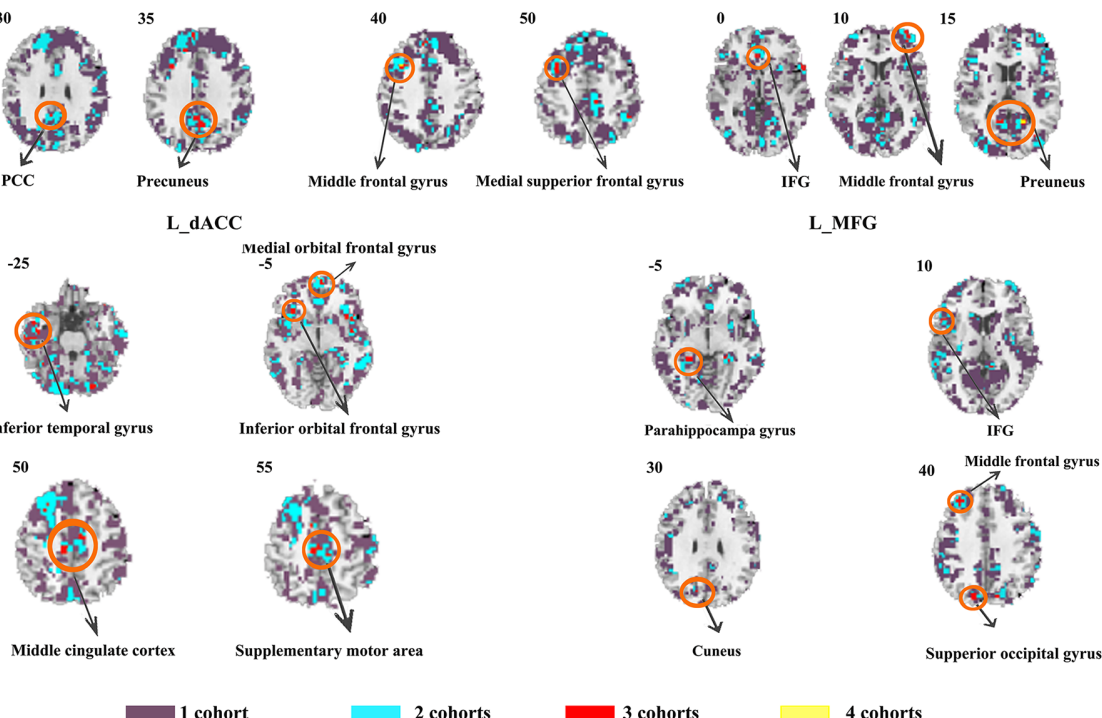

FIGURE 7 | The overlapped effect size results of the individual dataset in the ADHD combined subtypes. The threshold of effect size was set at 0.8 for each dataset. (A-G) indicate the results detected by using $R_{-} d A C C, R_{-} I F G, R \_M F G, L_{-} d A C C, L_{-} I F G$, and $L_{-} M F G$ as the seed regions. Purple indicates the regions detected only in one of the four datasets. Mint indicates the regions detected in two datasets. Red indicates the regions detected by only three datasets. Yellow indicates the regions detected in four datasets. ADHD, attention deficit hyperactivity disorder; dACC, dorsal anterior cingulate cortex; IFG, inferior frontal gyrus; MFG, middle frontal gyrus. 


\section{ADHD inattention subtype}

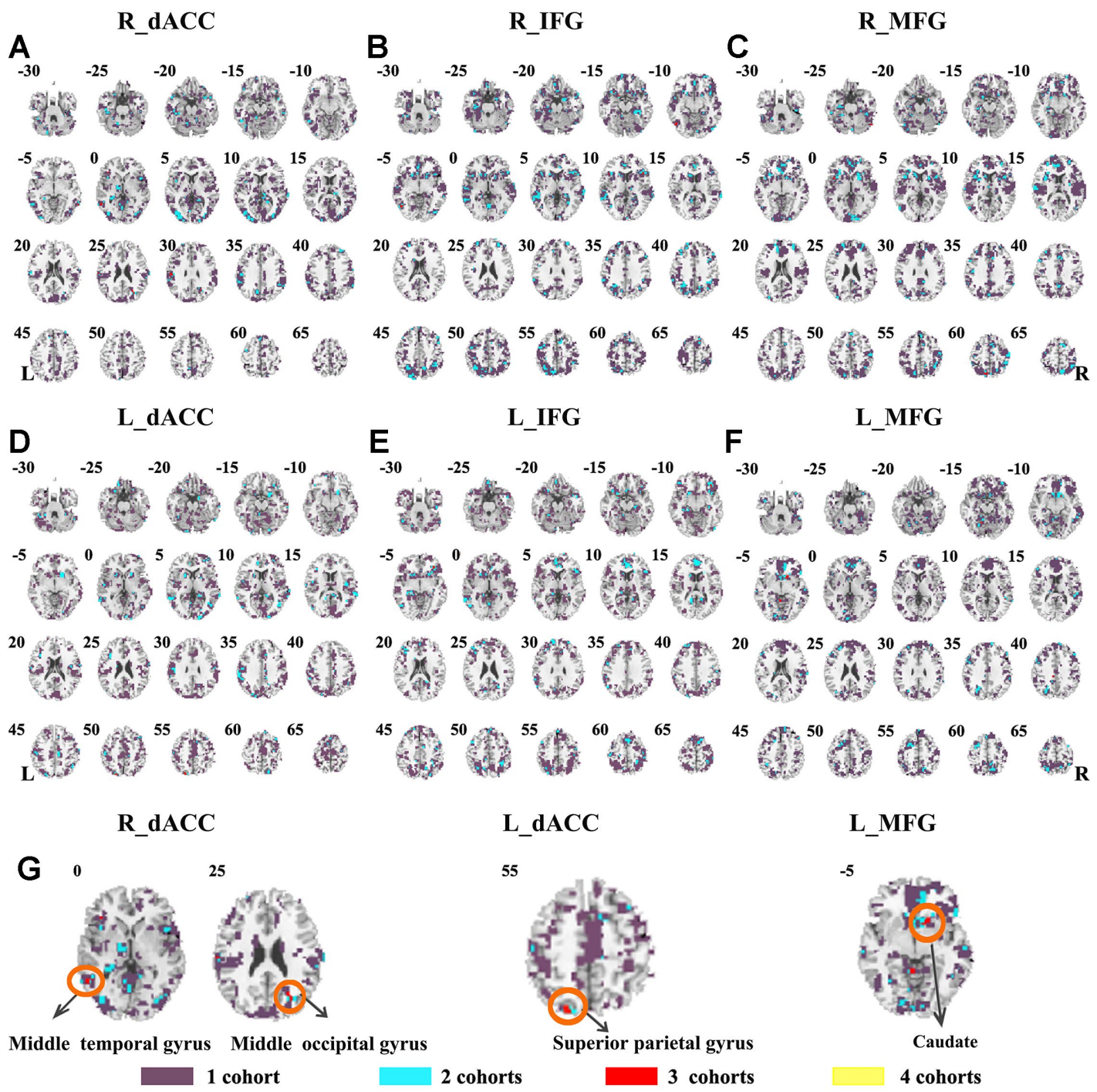

FIGURE 8 | The overlapped effect size results of the individual dataset in the ADHD inattention subtypes. The threshold of effect size was set at 0.8 for each dataset. (A-G) indicate the results detected by using R_dACC, R_IFG, R_MFG, L_dACC, L_IFG, and L_MFG as the seed regions. Purple indicates the regions detected only in one of the four datasets. Mint indicates the regions detected in two datasets. Red indicates the regions detected by only three datasets. Yellow indicates the regions detected in four datasets. ADHD, attention deficit hyperactivity disorder; dACC, dorsal anterior cingulate cortex; IFG, inferior frontal gyrus; MFG, middle frontal gyrus.

TABLE 4 | The statistical difference of the negative network connectivity between ADHD group and TDC group.

\begin{tabular}{lcccc}
\hline Dataset & $\begin{array}{c}\text { ADHD group } \\
\text { Mean } \pm \text { SD }\end{array}$ & $\begin{array}{c}\text { TDC group } \\
\text { Mean } \pm \text { SD }\end{array}$ & $\boldsymbol{t}$ & $\boldsymbol{p}$ \\
\hline NYU & $-1.31 \pm 0.40$ & $-1.45 \pm 0.31$ & 1.54 & 0.13 \\
PKU1 & $-1.51 \pm 0.21$ & $-1.60 \pm 0.20$ & 1.24 & 0.23 \\
PKU2 & $-1.30 \pm 0.26$ & $-1.41 \pm 0.27$ & 1.57 & 0.12 \\
PKU3 & $-1.30 \pm 0.30$ & $-1.36 \pm 0.28$ & 0.66 & 0.51 \\
\hline
\end{tabular}

No significant difference could be preserved after the multiple comparison correction. $A D H D$, attention deficit hyperactivity disorder; TDC, typical developing children.
Several limitations exist in the present study. First, we explored the functional connectivity with the seed regions of R_dACC, R_IFG, R_MFG, L_dACC, L_IFG, and L_MFG and the negative connectivity between ECN and DMN. Thus, our results were restricted to these measurements, which could not be extended to other regions and networks. Second, only ADHD combined subtype and inattention subtype were involved in the present study. We did not perform an analysis on the data of hyperactivity/impulsivity subtype because datasets of ADHD200 only include nine subjects with this subtype. Further studies recruiting subjects with hyperactivity/impulsivity are 


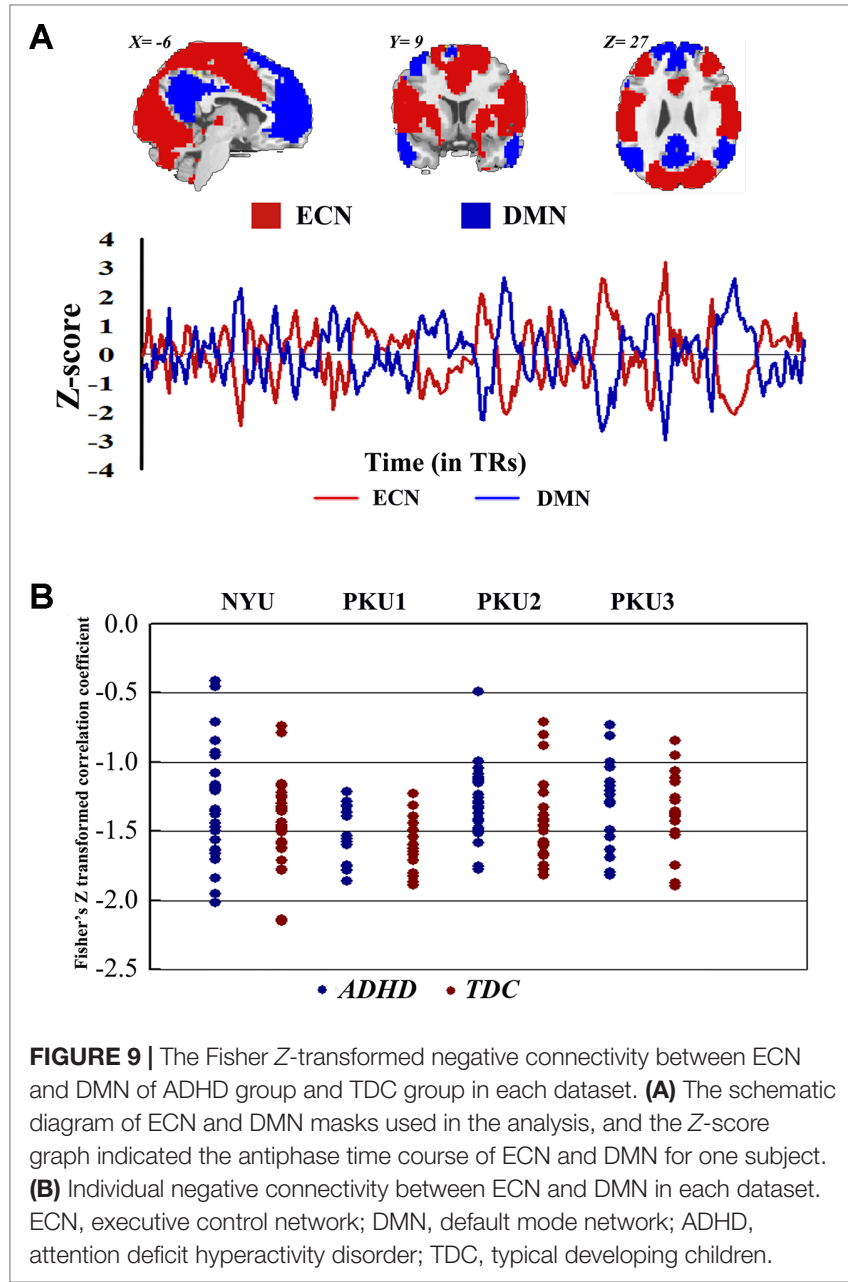

helpful for the validation of our findings. Lastly, we explored the contribution of different subtypes to the inconsistency in ADHD neuroimaging findings; however, the sample size for statistical analysis was too small. For example, PKU1 only included nine ADHD inattention subtype subjects and six ADHD combined subtype subjects. Their contribution should be further explored on a large sample dataset in the future.

\section{CONCLUSIONS}

Functional connectivity provided profound information for us to understand the pathological mechanism of ADHD. This is the first study, to our knowledge, to assess the consistency of abnormal functional connectivity in children with ADHD across different datasets. We found that the results of abnormal functional connectivity were inconsistent across datasets, even across three datasets from the same research site. And there was no significant difference between ADHD and TDC in the negative connectivity between ECN and DMN. More importantly, abnormal functional connectivity of the combined subtype was more consistent than that of the inattention subtype. These results provided methodological implications for the

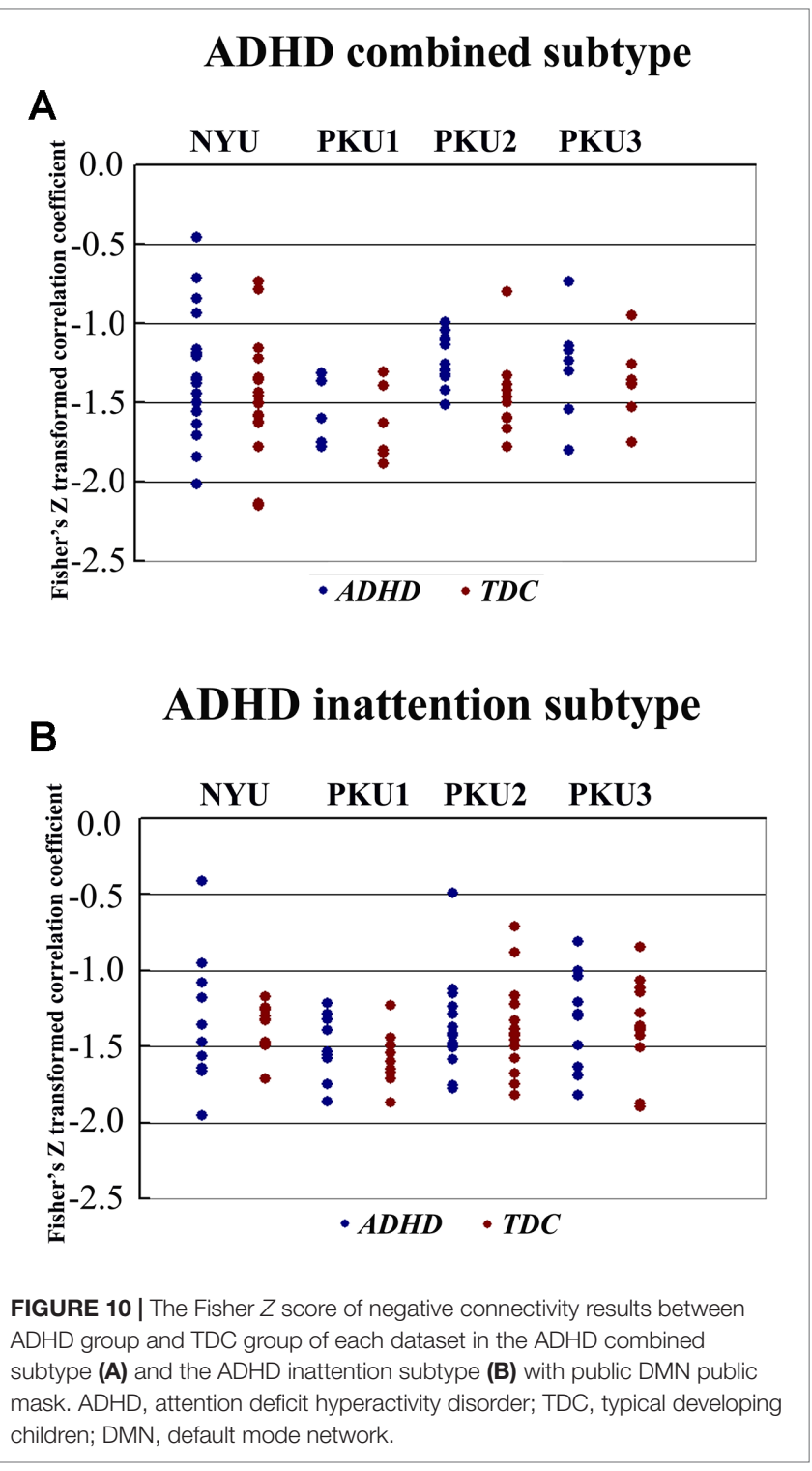

TABLE 5 | The statistical difference of the negative network connectivity between ADHD subtype group and TDC group.

\begin{tabular}{lcccc}
\hline Dataset & $\begin{array}{c}\text { ADHD group } \\
\text { Mean } \pm \text { SD }\end{array}$ & $\begin{array}{c}\text { TDC group } \\
\text { Mean } \pm \text { SD }\end{array}$ & $\boldsymbol{t}$ & $\boldsymbol{p}$ \\
\hline \multicolumn{4}{c}{ ADHD combined subtype } \\
NYU & $-1.30 \pm 0.39$ & $-1.49 \pm 0.36$ & 1.60 & 0.12 \\
PKU1 & $-1.53 \pm 0.21$ & $-1.64 \pm 0.24$ & 0.86 & 0.41 \\
PKU2 & $-1.23 \pm 0.16$ & $-1.46 \pm 0.25$ & 2.69 & 0.01 \\
PKU3 & $-1.28 \pm 0.33$ & $-1.37 \pm 0.24$ & 0.61 & 0.55 \\
& ADHD inattention subtype & & \\
NYU & $-1.33 \pm 0.44$ & $-1.38 \pm 0.16$ & 0.34 & 0.74 \\
PKU1 & $-1.50 \pm 0.22$ & $-1.58 \pm 0.18$ & 0.84 & 0.41 \\
PKU2 & $-1.36 \pm 0.30$ & $-1.38 \pm 0.29$ & 0.21 & 0.83 \\
PKU3 & $-1.31 \pm 0.30$ & $-1.36 \pm 0.31$ & 0.34 & 0.73 \\
\hline
\end{tabular}

No significant difference could be preserved after the multiple comparison correction. $A D H D$, attention deficit hyperactivity disorder; TDC, typical developing children. 
rs-fMRI studies of children with ADHD, and subtype should be involved in the analysis as a critical factor in the future studies.

\section{DATA AVAILABILITY STATEMENT}

Publicly available datasets were analyzed in this study. This data can be found here: http://fcon_1000.projects.nitrc.org/indi/ adhd 200 .

\section{ETHICS STATEMENT}

Signed informed consent was obtained from all participants or their legal guardian before participation.

\section{AUTHOR CONTRIBUTIONS}

$\mathrm{HZ}$ and Y-FZ conceived and designed the experiment. Z-WZ and X-QL performed the data analysis. Y-TF, HL, Q-JC, LS and

\section{REFERENCES}

1. Polanczyk GV, Salum GA, Sugaya LS, Caye A, Rohde LA. Annual research review: a meta-analysis of the worldwide prevalence of mental disorders in children and adolescents. J Child Psychol Psychiatry (2015) 56(3):345-65. doi: $10.1111 /$ jcpp. 12381

2. Castellanos FX, Sonuga-Barke EJS, Milham MP, Tannock R. Characterizing cognition in ADHD: beyond executive dysfunction. Trends Cogn Sci (2006) 10:117-23. doi: 10.1016/j.tics.2006.01.011

3. Bush G. Attention-deficit/hyperactivity disorder and attention networks. Neuropsychopharmacology (2010) 35:278-300. doi: 10.1038/npp.2009.120

4. Zang Y, He Y, Zhu C, Cao Q, Sui M, Liang M, et al. Altered baseline brain activity in children with ADHD revealed by resting-state functional MRI. Brain Dev (2007) 29:83-91. doi: 10.1016/j.braindev.2006.07.002

5. Zang Y, Jiang T, Lu Y, He Y, Tian L. Regional homogeneity approach to fMRI data analysis. Neuroimage (2004) 22:394-400. doi: 10.1016/j. neuroimage.2003.12.030

6. Buckner RL, Sepulcre J, Talukdar T, Krienen FM, Liu H, Hedden T, et al. Cortical hubs revealed by intrinsic functional connectivity: mapping, assessment of stability, and relation to Alzheimer's disease. J Neurosci (2009) 29:1860-73. doi: 10.1523/JNEUROSCI.5062-08.2009

7. Herz DM, Eickhoff SB, Lokkegaard A, Siebner HR. Functional neuroimaging of motor control in Parkinson's disease: a meta-analysis. Hum Brain Mapp (2014) 35:3227-37. doi: 10.1002/hbm.22397

8. Iwabuchi SJ, Krishnadas R, Li C, Auer DP, Radua J, Palaniyappan L. Localized connectivity in depression: a meta-analysis of resting state functional imaging studies. Neurosci Biobehav Rev (2015) 51:77-86. doi: 10.1016/j. neubiorev.2015.01.006

9. Wang X, Jiao Y, Tang T, Wang H, Lu Z. Altered regional homogeneity patterns in adults with attention-deficit hyperactivity disorder. Eur J Radiol (2013) 82(9):1552-7. doi: 10.1016/j.ejrad.2013.04.009

10. Cao Q, Zang Y, Sun L, Sui M, Long X, Zou Q, et al. Abnormal neural activity in children with attention deficit hyperactivity disorder: a restingstate functional magnetic resonance imaging study. NeuroReport (2006) 17(10):1033-6. doi: 10.1097/01.wnr.0000224769.92454.5d

11. Di Martino A, Zuo XN, Kelly C, Grzadzinski R, Mennes M, Schvarcz A, et al. Shared and distinct intrinsic functional network centrality in autism and attention-deficit/hyperactivity disorder. Biol Psychiatry (2013) 74(8):623-32. doi: 10.1016/j.biopsych.2013.02.011

12. Wang J, Zheng L, Cao Q, Wang Y, Sun L, Zang Y, et al. Inconsistency in abnormal brain activity across cohorts of ADHD-200 in children with
Y-FW provided advice on the analysis and interpretation of the results. Z-WZ and HZ wrote the paper.

\section{FUNDING}

This work is supported by the National Key R\&D Program of China (2018YFC1312600 and 2018YFC1312603), the National Natural Sciences Foundation of China (31471084, 81520108016, 81471382, 81873804, and 81771479), the National Basic Research Program of China (973 program 2014CB846104), and Zhejiang Fundamental Public Welfare Research Program (LGJ19C090001). HZ is supported by Scientific Research Staring Foundation from Hangzhou Normal University.

\section{SUPPLEMENTARY MATERIAL}

The Supplementary Material for this article can be found online at: https://www.frontiersin.org/articles/10.3389/fpsyt.2019.00692/ full\#supplementary-material attention deficit hyperactivity disorder. Front Neurosci (2017) 11320:1-10. doi: 10.3389/fnins.2017.00320

13. Friston KJ, Frith CD, Liddle PF, Frackowiak RSJ. Functional connectivity: the principal-component analysis of large (PET) data sets. J Cereb Blood Flow Metabol (1993) 13(1):5-14. doi: 10.1038/jcbfm.1993.4

14. Weissman DH, Roberts KC, Visscher KM, Woldorff MG. The neural bases of momentary lapses in attention. Nat Neurosci (2006) 9:971-8. doi: 10.1038/ nn1727

15. Castellanos FX, Margulies DS, Kelly C, Uddin LQ, Ghaffari M, Kirsch A, et al. Cingulate-precuneus interactions: a new locus of dysfunction in adult attention-deficit/hyperactivity disorder. Biol Psychiatry (2008) 63(3):332-7. doi: 10.1016/j.biopsych.2007.06.025

16. Sun L, Cao Q, Long X, Sui M, Cao X, Zhu C, et al. Abnormal functional connectivity between the anterior cingulate and the default mode network in drug-naive boys with attention deficit hyperactivity disorder. Psychiatry Res (2012) 201(2):120-7. doi: 10.1016/j.pscychresns.2011.07.001

17. Hong SB, Harrison BJ, Fornito A, Sohn CH, Song IC, Kim JW. Functional dysconnectivity of corticostriatal circuitry and differential response to methylphenidate in youth with attention-deficit/hyperactivity disorder. $J$ Psychiatry Neurosci (2015) 40(1):46-57. doi: 10.1503/jpn.130290

18. Raichle ME, Macleod AM, Snyder AZ, Powers WJ, Gusnard DA, Shulman GL. Inaugural article by a recently elected academy member: a default mode of brain function. Proc Natl Acad Sci U S A (2001) 98(2):676. doi: $10.1073 /$ pnas.98.2.676

19. Shulman GL, Corbetta M, Buckner RL, Fiez JA, Miezen FM, Raichle ME, et al. Common blood flow changes across visual tasks: I. Increases in subcortical structures and cerebellum but not in nonvisual cortex. J Cognit Neurosci (1997) 9:624-47. doi: 10.1162/jocn.1997.9.5.624

20. Gusnard DA, Raichle ME. Searching for a baseline: functional imaging and the resting human brain. Nat Rev Neurosci (2001) 2(10):685-94. doi: $10.1038 / 35094500$

21. Buckner RL, Andrews-Hanna JR, Schacter DL. The brain's default network: anatomy, function, and relevance to disease. Ann NY Acad Science (2008) 1124:1-38. doi: 10.1196/annals.1440.011

22. Naghavi HR, Nyberg L. Common fronto-parietal activity in attention, memory, and consciousness: shared demands on integration? Conscious Cogn (2005) 14(2):390-425. doi: 10.1016/j.concog.2004.10.003

23. Querne L, Fall S, Le Moing AG, Bourel-Ponchel E, Delignières A, Simonnot A, et al. Effects of methylphenidate on default-mode network/ task-positive network synchronization in children with ADHD. Eur J Paediatric Neurol (2013) 17:S95-5. doi: 10.1016/S1090-3798(13)70333-7 
24. Greicius MD, Krasnow B, Reiss AL, Menon V. Functional connectivity in the resting brain: a network analysis of the default mode hypothesis. Proc Natl Acad Sci (2003) 100(1):253-8. doi: 10.1073/pnas.0135058100

25. Fox MD, Snyder AZ, Vincent JL, Corbetta M, Van Essen DC, Raichle ME. From the cover: the human brain is intrinsically organized into dynamic, anticorrelated functional networks. Proc Natl Acad Sci (2005) 102(27):96738. doi: 10.1073/pnas.0504136102

26. ADHD-200-Consortium, T. The ADHD-200 consortium: a model to advance the translational potential of neuroimaging in clinical neuroscience. Front Syst Neurosci (2012) 6:1-5. doi: 10.3389/fnsys.2012.00062

27. Gadow KD, Devincent CJ, Pomeroy J. ADHD symptom subtypes in children with pervasive developmental disorder. J Autism Dev Disord (2006) 36(2):271-83. doi: 10.1007/s10803-005-0060-3

28. Brown MRG, Sidhu GS, Russell G, Nasimeh A, Meysam B, Silverstone PH, et al. ADHD-200 global competition: diagnosing ADHD using personal characteristic data can outperform resting state fMRI measurements. Front Syst Neurosci (2012) 6:1-22. doi: 10.3389/fnsys.2012.00069

29. Yang H, Wu Q, Guo L, Li Q, Long X, Huang X, et al. Abnormal spontaneous brain activity in medication-naive ADHD children: a resting state fMRI study. Neurosci Lett (2011) 502(2):89-93. doi: 10.1016/j.neulet.2011.07.028

30. Yan C, Zang Y. DPARSF: a MATLAB toolbox for "pipeline" data analysis of resting-state fMRI. Front Syst Neurosci (2010) 4:13. 1-7. doi: 10.3389/ fnsys. 2010.00013

31. Song X, Dong Z, Long X, Li S, Zuo X, Zhu C, et al. REST: a toolkit for restingstate functional magnetic resonance imaging data processing. PLoS ONE (2011) 6:e25031. doi: 10.1371/journal.pone.0025031

32. Tamm L, Menon V, Reiss AL. Parietal attentional system aberrations during target detection in adolescents with attention deficit hyperactivity disorder: event-related fMRI evidence. Am J Psychiatry (2006) 163(6):1033-43. doi: 10.1176/ajp.2006.163.6.1033

33. Kawohl W, Brühl A, Krowatschek G, Ketteler D, Herwig U. Functional magnetic resonance imaging of tics and tic suppression in Gilles de la Tourette syndrome. World J Biol Psychiatry (2009) 10(4-2):567-70. doi: $10.1080 / 15622970802118356$

34. Ge R, Torres I, Brown JJ, Gregory E, McLellan E, Downar JH, et al. Functional disconnectivity of the hippocampal network and neural correlates of memory impairment in treatment-resistant depression. J Affect Disord (2019) 253:248-56. doi: 10.1016/j.jad.2019.04.096

35. Kimura D. The asymmetry of the human brain. Sci Am (1973) 228(3):70-8. doi: 10.1038/scientificamerican0373-70

36. Watkins KE, Paus T, Lerch JP, Zijdenbos A, Collins DL, Neelin P, et al. Structural asymmetries in the human brain: a voxel-based statistical analysis of 142 MRI scans. Cer Cortex (2001) 11(9):868-77. doi: 10.1093/ cercor/11.9.868

37. Yeo BTT, Krienen FM, Sepulcre J, Sabuncu MR, Lashkari D, Hollinshead M, et al. The organization of the human cerebral cortex estimated by functional connectivity. J Neurophysiol (2011) 106(3):1125-65. doi: 10.1152/ jn.00338.2011

38. Uddin LQ, Kelly AMC, Biswal BB, Castellanos FX, Milham MP. Functional connectivity of default mode network components: correlation, anticorrelation, and causality. Hum Brain Mapp (2008) 30(2):625-37. doi: 10.1002/hbm.20531

39. Fox MD, Zhang D, Snyder AZ, Raichle ME. The global signal and observed anticorrelated resting state brain networks. J Neurophysiol (2009) 101(6):3270-83. doi: 10.1152/jn.90777.2008

40. Vincent JL, Kahn I, Snyder AZ, Raichle ME, Buckner RL. Evidence for a frontoparietal control system revealed by intrinsic functional connectivity. $J$ Neurophysiol (2008) 100(6):3328-42. doi: 10.1152/jn.90355.2008
41. Cohen J. Statistical power analysis for the behavioral sciences. New York, NY: Routledge (2013). doi: 10.4324/9780203771587

42. Dice LR. Measures of the amount of ecologic association between species. Ecology (1944) 26:297-302. doi: 10.2307/1932409

43. Bos DJ, Oranje B, Achterberg M, Vlaskamp C, Ambrosino S, Reus MA, et al. Structural and functional connectivity in children and adolescents with and without attention deficit/hyperactivity disorder. J Child Psychol Psychiatry (2017) 581:810-8. doi: 10.1111/jcpp.12712

44. Wang L, Zhu C, He Y, Zang Y, Cao Q, Zhang H, et al. Altered small-world brain functional networks in children with attention-deficit/hyperactivity disorder. Hum Brain Mapp (2008) 30(2):638-49. doi: 10.1002/hbm.20530

45. Fair DA, Posner J, Nagel BJ, Bathula D, Dias TGC, Mills KL, et al. Atypical default network connectivity in youth with attention-deficit/ hyperactivity disorder. Biol Psychiatry (2010) 68(12):1084-91. doi: 10.1016/j. biopsych.2010.07.003

46. Huang-Pollock CL, Mikami AY, Pfiffner L, Mcburnett K. ADHD subtype differences in motivational responsivity but not inhibitory control: evidence from a reward-based variation of the stop signal paradigm. J Clin Child Adolesc Psychol (2007) 36(2):127-36. doi: 10.1080/15374410701274124

47. Fransson P. Spontaneous low-frequency bold signal fluctuations: an fMRI investigation of the resting-state default mode of brain function hypothesis. Hum Brain Mapp (2010) 26(1):15-29. doi: 10.1002/hbm.20113

48. Golland Y, Golland P, Bentin S, Malach R. Data-driven clustering reveals a fundamental subdivision of the human cortex into two global systems. Neuropsychologia (2008) 46(2):540-53. doi: 10.1016/j. neuropsychologia.2007.10.003

49. Kelly AMC, Uddin LQ, Biswal BB, Castellanos FX, Milham MP. Competition between functional brain networks mediates behavioral variability. Neuroimage (2008) 39(1):527-37. doi: 10.1016/j.neuroimage.2007.08.008

50. Van Dijk KRA, Hedden T, Venkataraman A, Evans KC, Lazar SW, Buckner RL. Intrinsic functional connectivity as a tool for human connectomics: theory, properties, and optimization. J Neurophysiol (2010) 103(1):297-321. doi: $10.1152 /$ jn. 00783.2009

51. Maarten M, Natan VP, Clare K, Adriana DM, Xavier CF, Milham MP. Resting state functional connectivity correlates of inhibitory control in children with attention-deficit/hyperactivity disorder. Front Psychiatry (2012) 83(2):1-17. doi: $10.3389 /$ fpsyt.2011.00083

52. Duann JR, Ide JS, Luo X, Li CSR. Functional connectivity delineates distinct roles of the inferior frontal cortex and presupplementary motor area in stop signal inhibition. J Neurosci (2009) 29(32):10171-9. doi: 10.1523/ JNEUROSCI.1300-09.2009

53. Rubia K, Smith AB, Brammer MJ, Toone B, Taylor E. Abnormal brain activation during inhibition and error detection in medication-naive adolescents with ADHD. Am J Psychiatry (2005) 162(6):1067-75 doi: 10.1176/appi.ajp.162.6.1067.

Conflict of Interest: The authors declare that the research was conducted in the absence of any commercial or financial relationships that could be construed as a potential conflict of interest.

Copyright (๑) 2019 Zhou, Fang, Lan, Sun, Cao, Wang, Luo, Zang and Zhang. This is an open-access article distributed under the terms of the Creative Commons Attribution License (CC BY). The use, distribution or reproduction in other forums is permitted, provided the original author(s) and the copyright owner(s) are credited and that the original publication in this journal is cited, in accordance with accepted academic practice. No use, distribution or reproduction is permitted which does not comply with these terms. 